\title{
Adaptive Vectorial Filter for Grid Synchronization of Power Converters Under Unbalanced and/or Distorted Grid Conditions
}

\author{
Sergio Vazquez, Member, IEEE, Juan A. Sanchez, Manuel R. Reyes, J. I. Leon, Member, IEEE and J. M. \\ Carrasco, Member, IEEE
}

\begin{abstract}
This paper presents a new synchronization scheme for detecting multiple positive-/negative-sequence frequency harmonics in three-phase systems for grid-connected power converters. The proposed technique is called MAVF-FLL because it is based on the use of multiple adaptive vectorial filters (AVFs) working together inside a harmonic decoupling network (HDN), resting on a frequency-locked loop (FLL) which makes the system frequency adaptive. The method uses the vectorial properties of the three-phase input signal in the $\alpha \beta$ reference frame in order to obtain the different harmonic components. The MAVF-FLL is fully designed and analyzed addressing the tuning procedure in order to obtain the desired and predefined performance. The proposed algorithm is evaluated by both simulation and experimental results, demonstrating its ability to perform as required for detecting different harmonic components under a highly unbalanced and distorted input grid voltage.
\end{abstract}

\section{INTRODUCTION}

$\mathbf{T}$ HE DISTRIBUTED power generation networks and smartgrids are characterized by a high penetration of renewable energies, like wind or PV, and the inclusion of energy storage systems. The integration of these energy resources has been achieved by means of using power electronic devices as grid interface [1]-[3]. On the other hand, system operators have introduced new and high demanding grid codes oriented to ensure a proper performance of these devices in order to achieve a reliable, efficient and safe operation of the grid [4], [5]. As a consequence, companies and academia have paid a great effort to develop new control strategies for grid connected power converters. The new control schemes allow to achieve an optimal and efficient operation of power electronics converters [6]-[12].

The new grid codes impose to power converters to stay connected and supporting the grid service even if the voltage at its point of common coupling (PCC) is distorted and unbalanced. In this way, power converters should be properly synchronized with the grid. Therefore, the synchronization

Manuscript received September 06, 2012. Accepted for publication March 25, 2013. This work was supported by the Spanish Economy and Competitiveness under project ENE2012-36897. Copyright (C) 2013 IEEE. Personal use of this material is permitted. However, permission to use this material for any other purposes must be obtained from the IEEE by sending a request to pubspermissions@ieee.org. S. Vazquez, J.A. Sanchez, J.I. Leon and J.M. Carrasco are with the Electronic Engineering Department, University of Seville (Spain), (e-mail: sergi@us.es). M.R. Reyes is with the Power Electronics area at Win Inertia (Spain), (e-mail: manreyes@gmail.com). algorithm accuracy plays a fundamental role in the performance achieved by the control strategies. The main task of this mechanism is to provide synchronization with the voltage at the PCC under any circumstance. Several strategies can be used to synchronize the power converter with the grid voltage. The most conventional is the phase-locked loop (PLL) technology [13]-[15]. This technique has been extensively used in both single and three-phase systems, where it is based on synchronous reference frame (SRF-PLL) [16]. The main drawback of SRF-PLLs is that their performance under unbalanced and/or distorted voltage are reduced. This makes this technique unsuitable for the power converters under the new grid codes requirements [17].

Several proposals have been developed to cope with this issue such as the use of two SRFs and a decoupling network to separate the effects of the positive- and negative-sequence voltage components [18], or the use of three single-phase PLLs combined with a positive-sequence calculator without using any SRF [19].

Another approach consists of using the frequency-locking concept for synchronizing. The main advantage of the frequency-locked loop (FLL) is that it estimates the frequency of the input grid voltage, which is a signal that is not affected by sudden changes [20]. Therefore it is more convenient than the phase-angle based method used by the PLL algorithms. A system consisting of a synchronization scheme based on multiple second-order generalized integrator resting on an FLL (MSOGI-FLL) was presented in [21]. Its main advantage is that it allows to perform an analysis of the input signal in real-time, providing an accurate estimation of the fundamental and harmonic components of the grid voltage.

On the other hand, solutions based on the application of parallel adaptive notch filters (ANFs) for estimating the harmonic content in real-time can also be found in the literature. A single-phase scheme was presented in [22] and later was extended to three-phase systems by applying three single-phase synchronization schemes based on a direct frequency-adaptive estimation algorithm [23], [24]. Other synchronization methods are also presented in the literature, like a delayed signal cancellation scheme shown in [25] or the use of vectorial filters as a synchronization scheme proposed in [26]-[28], where, complex coefficient band-pass filters are used to extract the positive-/negative-sequence frequency harmonics.

This paper presents a new synchronization scheme for 
detecting multiple positive-/negative-sequence frequency harmonics in three-phase systems. The proposed technique has been named MAVF-FLL because it is based on the use of multiple adaptive vectorial filters (AVFs). These AVFs have been used previously for controlling active filters with excellent results [29]. In the proposed synchronization approach, the AVFs work together inside a harmonic decoupling network (HDN), resting on an FLL.

Comparing the proposed AVF with MSOGI-FLL, the main difference is the way used to arrive to the AVF expression. The new method uses a vectorial approach where the $\beta$-axis measurement is influencing the filtered $\alpha$-axis signal and vice versa. On the other hand, the MSOGI-FLL is rather a particular band-pass filter that can be applied to single-phase, as well as three-phase signals represented in any reference frame (natural abc frame or the $\alpha \beta$ frame). In the three-phase case, the vectorial properties of the signals are then not considered until the filtered signals are used for sequence separation. The AVF presented in this paper is however inherently connected to the vectorial properties of three-phase signals in the $\alpha \beta$ reference frame, and is therefore specifically adapted to three-phase systems. Although it is possible to use it also for single-phase systems by considering these cases as a highly unbalanced three-phase system [26].

When brought into comparison with the cascaded delay signal cancellation PLL method [25], the proposed AVF strategy uses complex coefficient filters for grid synchronization. While in [25] it is applied the symmetric component theory to extract the fundamental-frequency positive sequence by employing finite impulse response (FIR) filters to attenuate the rest.

On the other hand, compared with previous complex coefficient filters used for positive-/negative-sequence harmonic detection, [26]-[28], the approach followed to obtain the AVF coefficients uses the vectorial properties of the threephase input signal in the $\alpha \beta$ reference frame in order to obtain the final complex coefficient filter. In this way, the proposed scheme lies on using a vectorial approach which is different from previous methods, because the expression for the synchronization algorithm is derived from the vector properties of the input signals providing useful characteristics for the tuning of the design constants. The main advantage of the method is that these constants can be chosen to provide a desired and predefined performance. Besides, the presented scheme can be easily implemented in low-cost DSP hardware with low computational cost permitting to estimate in real time the fundamental and harmonic components of the input signal.

The paper is divided as follows. In section II the AVF is derived. Then, in section III its functional block is analyzed and the criteria for tuning the design constants are established. After that, the frequency adaptation loop is proposed and analyzed in sections IV and V. The performance under unbalanced and distorted input voltage is studied in sections VI and VII. Finally section VIII shows the experimental results of the proposed algorithm in order to evaluate the effectiveness of the proposal when it is implemented in a real system based on a DSP platform.

\section{The Adaptive Vectorial Filter}

In this section, the adaptive vectorial filter (AVF) is designed. In order to develop the AVF lets suppose an input vector $a_{\alpha \beta}$ defined in the stationary reference frame $\alpha \beta$ by

$$
a_{\alpha \beta}=A_{p}\left\{\begin{array}{l}
\cos (\omega t) \\
\sin (\omega t)
\end{array}\right\} \text {. }
$$

Lets assume parameter $\omega$ is a constant or slowly variant value. This assumption can be considered true for reconstructing the grid voltages, because the grid frequency can not change suddenly. Thus the following analysis is a valid approximation for this application. In that case, it is possible to calculate the derivative over the time of $a_{\alpha \beta}$ as

$$
\frac{d a_{\alpha \beta}}{d t}=\omega J a_{\alpha \beta}
$$

where matrix $J$ is

$$
J=\left\{\begin{array}{rr}
0 & -1 \\
1 & 0
\end{array}\right\} .
$$

Lets $b_{\alpha \beta}$ be the output vector of the AVF. Then the scalar and vectorial products of $a_{\alpha \beta}$ and $b_{\alpha \beta}$ are

$$
\begin{aligned}
& s p=b_{\alpha \beta}^{T} a_{\alpha \beta} \\
& v p=b_{\alpha \beta}^{T} J a_{\alpha \beta} .
\end{aligned}
$$

From (4) and (5) it can be obtained the derivative over the time of $s p$ and $v p$

$$
\begin{aligned}
& \frac{d s p}{d t}=\left(\frac{d b_{\alpha \beta}^{T}}{d t}+\omega b_{\alpha \beta}^{T} J\right) a_{\alpha \beta} \\
& \frac{d v p}{d t}=\left(\frac{d b_{\alpha \beta}^{T}}{d t}+\omega b_{\alpha \beta}^{T} J\right) J a_{\alpha \beta} .
\end{aligned}
$$

As the objective is to reconstruct $a_{\alpha \beta}$ from $b_{\alpha \beta}$ then it must be satisfied that

$$
\begin{aligned}
& s p \quad \rightarrow \quad s p^{*}=a_{\alpha \beta}^{T} a_{\alpha \beta} \\
& v p \quad \rightarrow \quad v p^{*}=0 .
\end{aligned}
$$

Taking into account (8) and (9), equations (6) and (7) can be manipulated to define the expression of $b_{\alpha \beta}$ in such a way that it achieves these objectives.

If $\tilde{s p}$ is defined as

$$
\tilde{s p} \triangleq s p-s p^{*}
$$

then (6) can be transformed in

$\frac{d \tilde{s p}}{d t}+k_{s p} \tilde{s p}+\frac{d s p^{*}}{d t}-k_{s p} \tilde{s p}=\left(\frac{d b_{\alpha \beta}^{T}}{d t}+\omega b_{\alpha \beta}^{T} J\right) a_{\alpha \beta}$.

From (11) it can be noticed that choosing

$$
\frac{d s p^{*}}{d t}-k_{s p} \tilde{s p} \triangleq\left(\frac{d b_{\alpha \beta}^{T}}{d t}+\omega b_{\alpha \beta}^{T} J\right) a_{\alpha \beta},
$$

then 


$$
\frac{d \tilde{s p}}{d t}+k_{s p} \tilde{s p}=0
$$

It can be observed that (13) represents a first order linear system and that $\tilde{s p}$ tends exponentially towards zero when $k_{s p}>0$. Thus, condition represented in (8) is fulfilled.

Equation (12) can be simplified regarding (4) and that $s p^{*}$ is a constant. In that case, (12) is reduced to

$$
k_{s p} a_{\alpha \beta}^{T} a_{\alpha \beta}=\left(\frac{d b_{\alpha \beta}^{T}}{d t}+\omega b_{\alpha \beta}^{T} J+k_{s p} b_{\alpha \beta}^{T}\right) a_{\alpha \beta},
$$

therefore, the dynamics representing $b_{\alpha \beta}$ must satisfy

$$
\frac{d b_{\alpha \beta}^{T}}{d t}+\omega b_{\alpha \beta}^{T} J+k_{s p} b_{\alpha \beta}^{T}=k_{s p} a_{\alpha \beta}^{T}+k_{1} a_{\alpha \beta}^{T} J .
$$

Similar analysis can be done starting from (7). In that case $\tilde{v p}$ is defined as

$$
\tilde{v p} \triangleq v p-v p^{*},
$$

and therefore (7) can be manipulated to be

$$
\frac{d \tilde{v p}}{d t}+k_{v p} \tilde{v p}+\frac{d v p^{*}}{d t}-k_{v p} \tilde{v p}=\left(\frac{d b_{\alpha \beta}^{T}}{d t}+\omega b_{\alpha \beta}^{T} J\right) J a_{\alpha \beta} .
$$

Now if it is chosen

$$
\frac{d v p^{*}}{d t}-k_{v p} \tilde{v p} \triangleq\left(\frac{d b_{\alpha \beta}^{T}}{d t}+\omega b_{\alpha \beta}^{T} J\right) J a_{\alpha \beta}
$$

then

$$
\frac{d \tilde{v p}}{d t}+k_{v p} \tilde{v p}=0
$$

Again, it can be observed that (19) corresponds to a first order linear system with $\tilde{v p}$ tending exponentially towards zero when $k_{v p}>0$. Therefore condition in (9) is also achieved.

Now (18) can be simplified considering (5) and that $v p^{*}$ is a constant equal to 0 . Taking into account these factors, (18) can be rewritten as

$$
0=\left(\frac{d b_{\alpha \beta}^{T}}{d t}+\omega b_{\alpha \beta}^{T} J+k_{v p} b_{\alpha \beta}^{T}\right) J a_{\alpha \beta}
$$

and as a consequence the dynamics of $b_{\alpha \beta}$ should be defined as

$$
\frac{d b_{\alpha \beta}^{T}}{d t}+\omega b_{\alpha \beta}^{T} J+k_{v p} b_{\alpha \beta}^{T}=k_{2} a_{\alpha \beta}^{T} .
$$

Notice that conditions (8) and (9) should be satisfied simultaneously. In order to achieve this, design constants $k_{s p}, k_{1}$, $k_{v p}$ and $k_{2}$ should be chosen suitably. In this case, the choice for these constants is

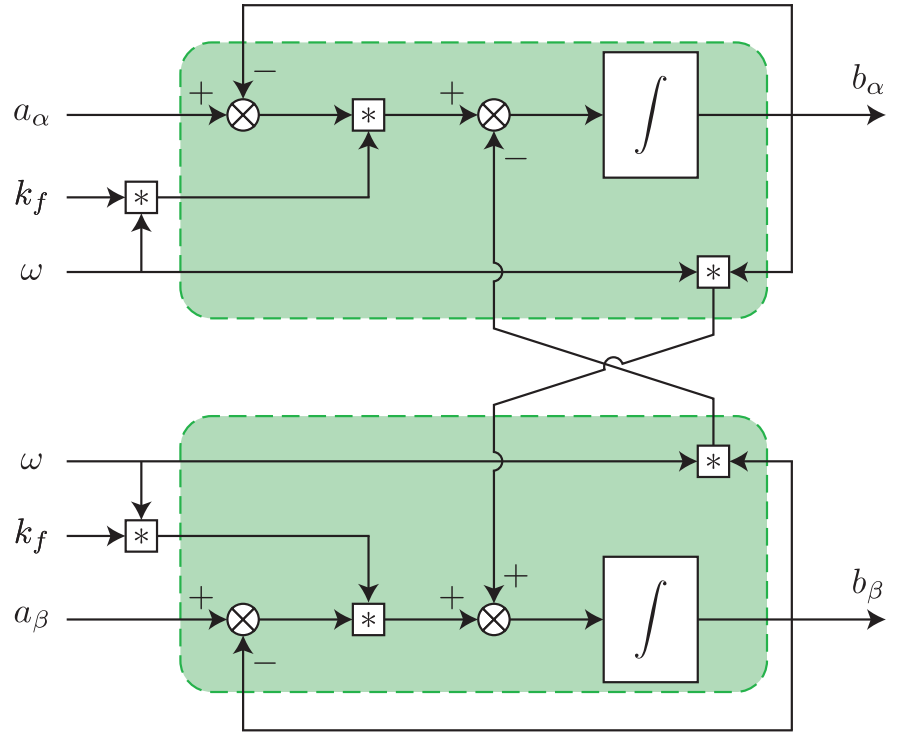

Fig. 1. Block diagram of the AVF structure (scalar notation).

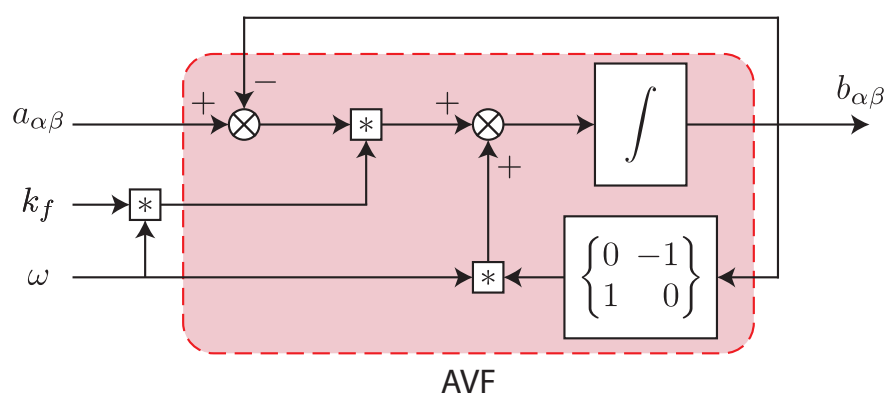

Fig. 2. Block diagram of the AVF structure (vectorial notation).

$$
\begin{aligned}
k_{s p} & =k_{v p}=k_{2}=\omega k_{f} \\
k_{1} & =0 .
\end{aligned}
$$

Under this situation (15) and (21) lead to the same equation

$$
\frac{d b_{\alpha \beta}}{d t}=\omega J b_{\alpha \beta}+\omega k_{f}\left(a_{\alpha \beta}-b_{\alpha \beta}\right),
$$

that finally represents the dynamic of $b_{\alpha \beta}$. It should be noticed that (24) is used to reconstruct an input signal of frequency $\omega$. In this way, if $\omega$ is considered as an unknown parameter that can be calculated in real time, then (24) can be made adaptive as will be shown in section IV. The value of the output signal can be calculated using the block diagram presented in Fig. 1 that has been expressed in its scalar form. As can be observed, the coupling between the $\alpha$ and $\beta$ axes is presented because the $\beta$-axis signals are influencing the filtered $\alpha$-signal and vice versa. This block diagram can be drawn in a compact way by using a vectorial notation as shown in Fig. 2.

\section{ANALysis And TUNing OF THE AVF}

To tune the design constant $k_{f}$ of the AVF, it is necessary to consider the final closed loop expressions for (13) and (19) when $k_{s p}$ and $k_{v p}$ are chosen equal to $\omega k_{f}$. Assuming $\omega$ to 


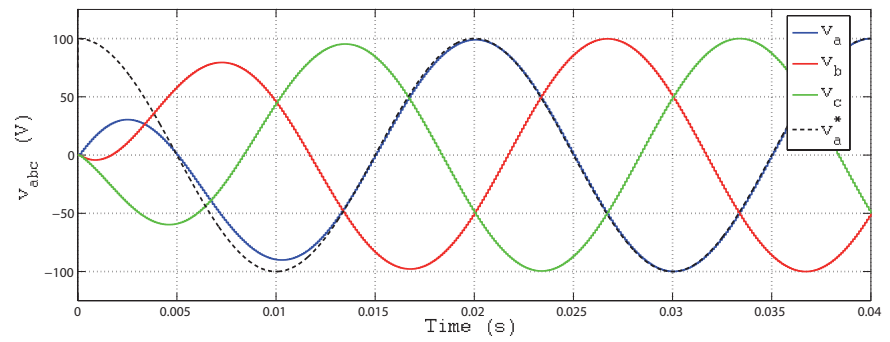

Fig. 3. Response of the AVF for a $50 \mathrm{~Hz}$ input signal.

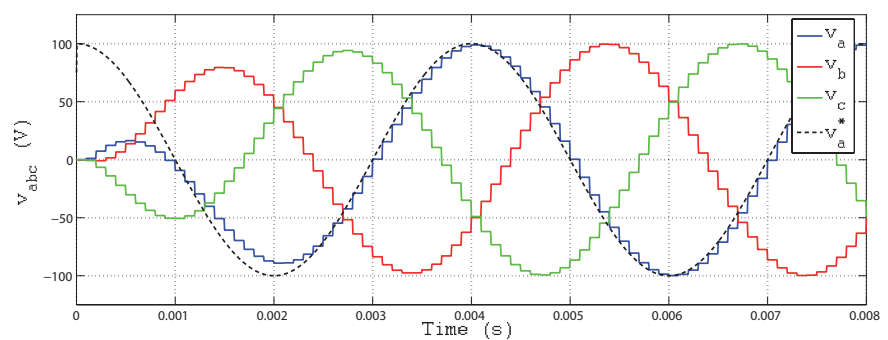

Fig. 4. Response of the AVF for a $250 \mathrm{~Hz}$ input signal.

be constant or slowly variant, it is possible to solve these equations.

$$
\begin{aligned}
\tilde{s p} & =s \tilde{p}_{o} e^{-\omega k_{f} t} \\
\tilde{v p} & =v \tilde{p}_{o} e^{-\omega k_{f} t}
\end{aligned}
$$

The value $k_{f}$ then can be chosen for a certain predefined settling time $T_{s}$. For instance, if in $T_{s}$ it is desired to have only a $1 \%$ of the initial error value then

$$
k_{f} \approx \frac{4.6}{\omega T_{s}} .
$$

Besides, $T_{s}$ can be selected proportional to the number of periods of the input signal that should be used to reconstruct the signal. For instance if parameter $k$ is chosen as the number of periods with $k>0$ then

$$
T_{s}=k T_{0},
$$

finally, considering that

$$
\omega=\frac{2 \pi}{T_{0}},
$$

then the design constant can be calculated as

$$
k_{f} \approx \frac{0.73}{k}
$$

Fig. 3 and Fig. 4 show the response of the AVF when the input signal frequency is $50 \mathrm{~Hz}$ and $250 \mathrm{~Hz}$ respectively with an amplitude of $100 \mathrm{~V}$ in both cases. The simulations have been performed using a discrete implementation of (24). The sampling frequency is $10 \mathrm{kHz}$ and the number of periods to reconstruct the input signal is chosen to be $k=1$ thus the value of $k_{f}$ is finally 0.73 . It can be noticed that in both cases

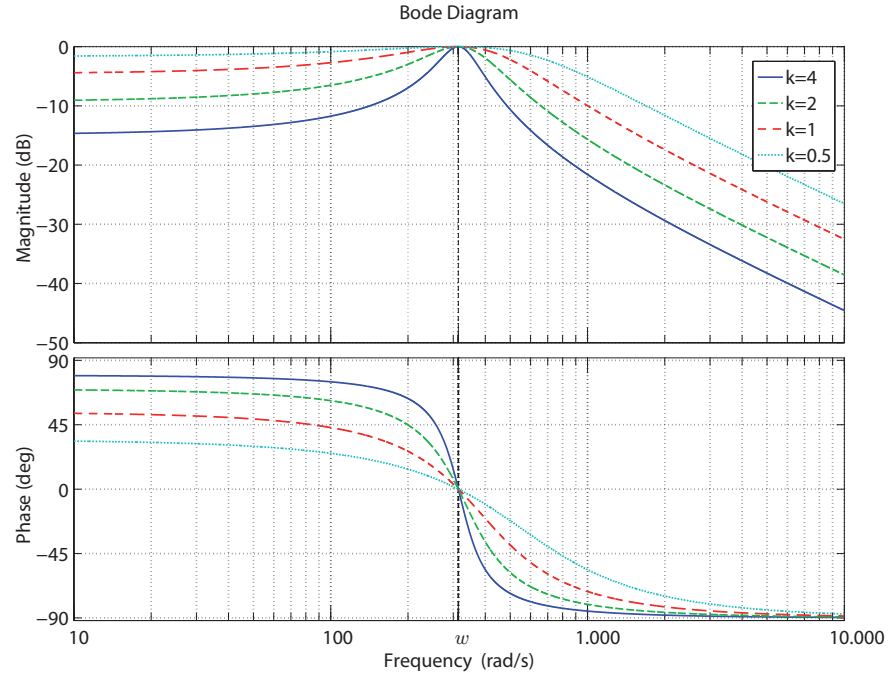

Fig. 5. Bode diagram of the AVF for $\omega$ equal to $50 \mathrm{~Hz}$ and different values of $k$.

the input signal is reconstructed properly and in one period as expected. However, the effect of the sampling frequency is clearly visible in Fig. 4. Thus, the sampling frequency should be chosen carefully when it is necessary to reconstruct high frequency signals.

The analysis of the behavior of the AVF can be done through its transfer function. To obtain such a transfer function, (24) should be expressed in a more suitable form.

$$
\left\{\begin{array}{l}
b_{\alpha} \\
b_{\beta}
\end{array}\right\}=\frac{\omega k_{f}}{\left(s+\omega k_{f}\right)^{2}+\omega^{2}}\left\{\begin{array}{cc}
\left(s+\omega k_{f}\right) & -\omega \\
\omega & \left(s+\omega k_{f}\right)
\end{array}\right\}\left\{\begin{array}{l}
a_{\alpha} \\
a_{\beta}
\end{array}\right\},
$$

now, using complex notation and considering

$$
\begin{gathered}
A_{\alpha \beta}=a_{\alpha}+j a_{\beta} \\
B_{\alpha \beta}=b_{\alpha}+j b_{\beta}
\end{gathered}
$$

the transfer function can be obtained from (31) like

$$
H(s)=\frac{B_{\alpha \beta}}{A_{\alpha \beta}}=\frac{\omega k_{f}\left(s+\omega k_{f}+j \omega\right)}{\left(s+\omega k_{f}\right)^{2}+\omega^{2}} .
$$

Fig. 5 shows the bode diagram of (34). In this diagram, $\omega$ has been chosen to be $50 \mathrm{~Hz}$, because this value is commonly adopted as the grid frequency in Europe. Other value could be adopted and the same conclusions would be addressed. Several plots have been represented for different values of parameter $k$, which finally defines the AVF design constant $k_{f}$ through (30). For any value of $k$, the gain and phase of the AVF for the frequency $\omega$ are zero. For any other frequency the input signal is attenuated and shifted. This behavior ensures the extraction of the desired component. Besides, it can be noticed that increasing $k$ the filter is more selective. However $k$ represents the number of cycles needed to reconstruct the desired frequency component. Thus a tradeoff between selectivity and speed should be considered when the input signal contents more than one harmonic component. 


\section{FREQUENCY AdAPTATION LoOP}

The frequency parameter $\omega$ of the AVF is critical to achieve the correct reconstruction of the input signal. As it can be observed in Fig. 5, if the input signal has a different frequency than the AVF parameter then this signal will be attenuated and therefore the output signal will never achieve the desired value. For this reason, it is necessary to develop a frequency adaptation loop allowing to calculate the correct parameter $\omega$. This task can be performed by a FLL which allow to calculate the actual value of $\omega$ [20], [21], [30].

Lets suppose that the frequency parameter of the AVF is $\widehat{\omega}$. Under this assumption (24) is transformed in

$$
\frac{d b_{\alpha \beta}}{d t}=\widehat{\omega} J b_{\alpha \beta}+\widehat{\omega} k_{f}\left(a_{\alpha \beta}-b_{\alpha \beta}\right) .
$$

When (35) is used to obtain the output signal, by introducing it in (17), the equation of the dynamic for $\tilde{v p}$ changes to

$$
\frac{d \tilde{v p}}{d t}+k_{f} \widehat{\omega} \tilde{v p}=s p \widetilde{\omega}
$$

where $\widetilde{\omega}$ is the error between the input signal frequency $\omega$ and $\widehat{\omega}$, defined as

$$
\widetilde{\omega} \triangleq \widehat{\omega}-\omega
$$

To estimate $\widehat{\omega}$ a Lyapunov function approach is used [31]. Lets define the positive semidefinite function $W$

$$
W=\frac{1}{2} \tilde{v p^{2}}+\frac{1}{2 \gamma} \widetilde{\omega}^{2}
$$

where $\gamma>0$ is the design constant used in the proposed adaptive law. The time derivative of (38) along the trajectories of $\tilde{v p}$ is

$$
\dot{W}=\tilde{v p}\left(s p \widetilde{\omega}-k_{f} \widehat{\omega} \tilde{v p}\right)+\frac{1}{\gamma} \widetilde{\omega} \frac{d \widetilde{\omega}}{d t},
$$

which is made negative semidefinite by proposing the adaptive law

$$
\frac{d \widetilde{\omega}}{d t}=-\gamma s p \tilde{v p}
$$

This yields

$$
\dot{W}=-k_{f} \widehat{\omega} \tilde{v p}{ }^{2}
$$

The stability and convergence of the proposed adaptive law can be done using Lasalle's arguments. As $W \geq 0$ and $\dot{W} \leq 0$ then should be true that

$$
\lim _{t \rightarrow \infty} \dot{W}=0,
$$

taking into account expression (41) and that $k_{f} \neq 0$ and $\widehat{\omega} \neq 0$, the only possibility is

$$
\lim _{t \rightarrow \infty} \tilde{v p}=0 \text {. }
$$

Finally, regarding (36) and considering that $s p \neq 0$, then

$$
\lim _{t \rightarrow \infty} \widetilde{\omega}=0 \text {. }
$$

Thus (44) ensures that the error between $\omega$ and $\widehat{\omega}$ tends to 0 and therefore that AVF can be properly tuned to the input signal frequency. To calculate $\widehat{\omega}$ it is necessary to consider that $\omega$ is a constant or slowly variant signal. Under this assumption

$$
\frac{d \widetilde{\omega}}{d t} \approx \frac{d \widehat{\omega}}{d t}
$$

So the final expression to calculate $\widehat{\omega}$ is

$$
\frac{d \widehat{\omega}}{d t}=-\gamma s p \tilde{v p}
$$

It should be noticed that if the input signal has a very high content of negative-sequence or any other harmonic component, the angular speed of the input vector may oscillate and the amplitude of these oscillations may be high. Therefore under this situation the last assumption will not be valid and these variations should be considered to calculate $\omega$. This situation only appears for very severe grid faults and in this case the power converter should be disconnected from the grid for a safe operation [32]. In spite of this, for the grid synchronization application it should be noticed that $\omega$ is a signal that does not suffer sudden variations during normal grid operation or under most of grid faults. Thus the proposed analysis can be considered as valid.

\section{ANALYSis AND TUNING OF THE FREQUENCY ADAPTATION LOOP}

Tuning the design constant $\gamma$ is not immediate due to the fact that the proposed adaptive law has a highly non linear behavior. As a first approximation considering definitions (4), (5) and (16) and that $v p^{*}=0$ then

$$
\begin{gathered}
s p=\left|a_{\alpha \beta}\right|\left|b_{\alpha \beta}\right| \cos \theta \\
\tilde{v p}=\left|a_{\alpha \beta}\right|\left|b_{\alpha \beta}\right| \sin \theta
\end{gathered}
$$

where $\theta$ is the angle between the input and output vector.

Lets rewrite the dynamic of $\widetilde{\omega},(40)$, as

$$
\frac{d \widetilde{\omega}}{d t}=-\frac{\xi}{\left|a_{\alpha \beta}\right|^{2}\left|b_{\alpha \beta}\right|^{2}} s p \tilde{v p}
$$

where $\xi$ is the new design constant. The main advantage of this formulation is that the behavior of (49) will not depend on the module values of the input and output vectors.

To calculate the value of $\xi$ it can be considered that when the system is reaching its steady state it can be assumed that

$$
\begin{aligned}
& s p \approx\left|a_{\alpha \beta}\right|\left|b_{\alpha \beta}\right| \\
& v p \approx \frac{\left|a_{\alpha \beta}\right|\left|b_{\alpha \beta}\right|}{k_{f} \widehat{\omega}_{\infty}} \widetilde{\omega},
\end{aligned}
$$

where $\widehat{\omega}_{\infty}$ is the value achieved by the estimator in steady state and (51) can be derived from (36).

Replacing (50) and (51) in (49) allows to approximate the behavior of the dynamic of $\widetilde{\omega}$ as

$$
\frac{d \widetilde{\omega}}{d t}=-\frac{\xi}{k_{f} \widehat{\omega}_{\infty}} \widetilde{\omega}
$$

This expression corresponds to a first order linear system that can be easily integrated as

$$
\widetilde{\omega}=\widetilde{\omega}_{o} e^{-\frac{\xi}{k_{f} \omega_{\infty}} t}
$$




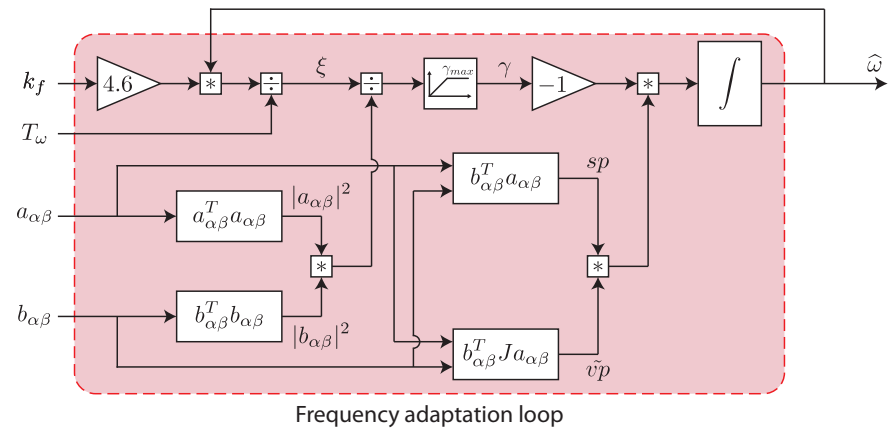

Fig. 6. Block diagram of the frequency adaptation loop.
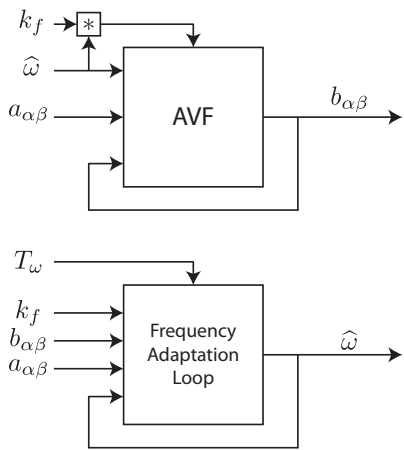

Fig. 7. Basic synchronization system scheme.

From (53) it is possible to calculate $\xi$ to achieve a desired settling time $T_{\omega}$. For instance, if for $T_{\omega}$ it is desired to have only a $1 \%$ of the initial error value then

$$
\xi \approx \frac{4.6 k_{f} \widehat{\omega}_{\infty}}{T_{\omega}}
$$

Now, considering (45) and replacing $\widehat{\omega}_{\infty}$ by $\widehat{\omega}$ in (54). The expressions used to estimate the AVF frequency parameter are

$$
\begin{aligned}
\xi & \triangleq \frac{4.6 k_{f} \widehat{\omega}}{T_{\omega}} \\
\frac{d \widehat{\omega}}{d t} & =-\frac{\xi}{\left|a_{\alpha \beta}\right|^{2}\left|b_{\alpha \beta}\right|^{2}} s p \tilde{v p} .
\end{aligned}
$$

Comparing (46) and (56) it is clear the gain $\gamma$ of the frequency adaptation loop is given by

$$
\gamma=\frac{\xi}{\left|a_{\alpha \beta}\right|^{2}\left|b_{\alpha \beta}\right|^{2}},
$$

where, $\xi$ is calculated by means of (55). It should be noticed that when the grid voltage is approaching to zero design constant $\gamma$ has to be limited to a maximum value [33].

Fig. 6 presents the block diagram of the frequency adaptation loop. Besides, Fig. 7 shows the scheme of the proposed synchronization system including the AVF and the frequency adaptation loop.

Fig. 8 and Fig. 9 show the performance achieved by the proposed frequency adaptation loop. In Fig. 8 the frequency of the input signal is changed suddenly from $50 \mathrm{~Hz}$ to $55 \mathrm{~Hz}$ and after $0.15 \mathrm{~s}$ it is restored to its previous value. A similar situation is presented in Fig. 9 but here the frequency step is from $50 \mathrm{~Hz}$ to $45 \mathrm{~Hz}$ and backward. The response of the proposed adaptive law is shown for several values of settling

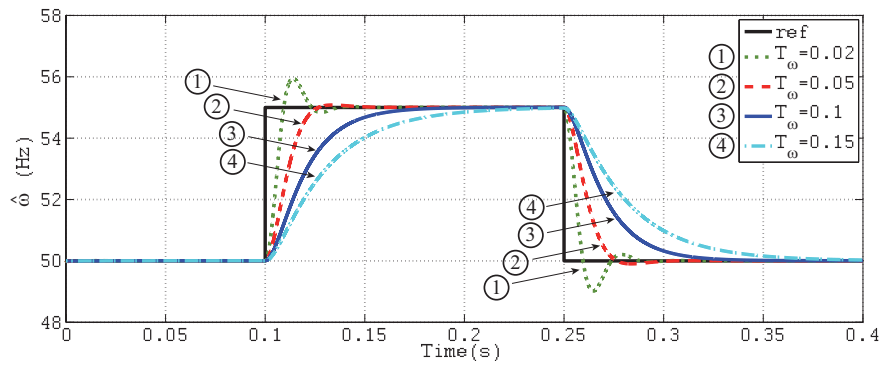

Fig. 8. Frequency adaptation loop response for an input signal frequency step from $50 \mathrm{~Hz}$ to $55 \mathrm{~Hz}$ and backward with different values of $T_{\omega}$.

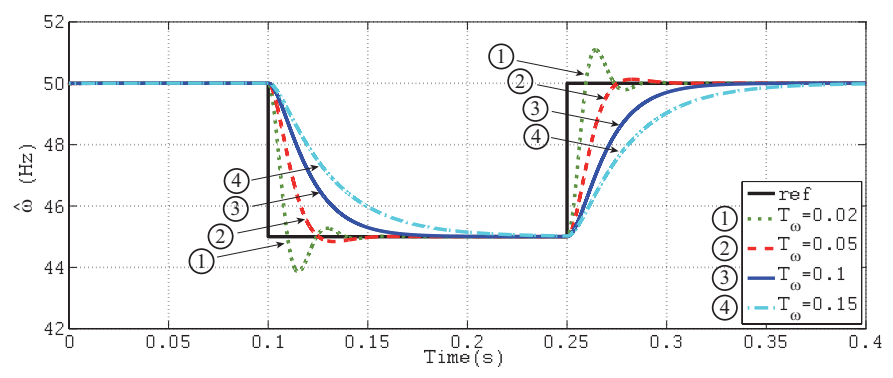

Fig. 9. Frequency adaptation loop response for an input signal frequency step from $50 \mathrm{~Hz}$ to $45 \mathrm{~Hz}$ and backward with different values of $T_{\omega}$.

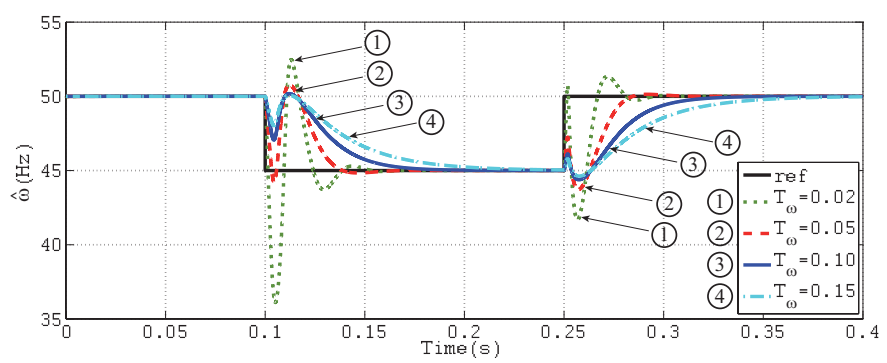

Fig. 10. Frequency adaptation loop response for an input signal frequency step from $50 \mathrm{~Hz}$ to $45 \mathrm{~Hz}$ and backward with different values of $T_{\omega}$.

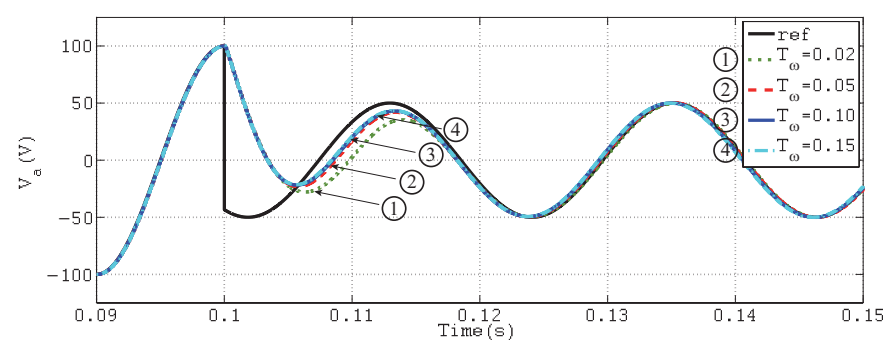

Fig. 11. AVF response for an input signal frequency step from $50 \mathrm{~Hz}$ to $45 \mathrm{~Hz}$ and backward with different values of $T_{\omega}$.

time. As it is shown, the proposed frequency adaptation loop performs as expected and the frequency estimation is achieved in the defined time interval.

It can be noticed in Fig. 8 and Fig. 9 that for $T_{\omega}$ in the range as the settling time of the AVF both dynamics interact and an overshoot appears. It should be considered that (53) is an approximation for the steady state behavior but the actual dynamic of $\widetilde{\omega}$ during transient is defined by (49) which has a non linear behavior, therefore there is a trade-off between settling time and overshoot. This must be considered when $T_{\omega}$ 


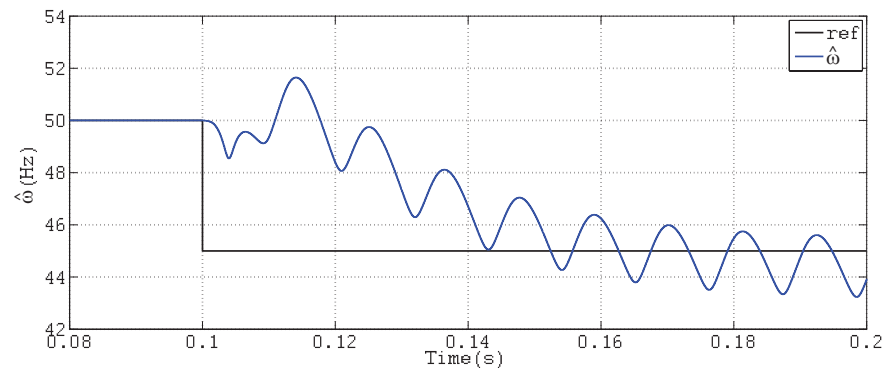

(a)

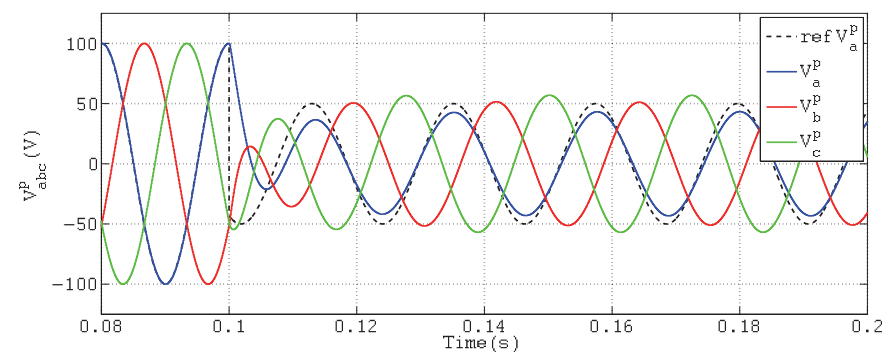

(b)

Fig. 12. System response: (a) Estimated frequency, (b) Positive output voltages.

is chosen, especially if not only a frequency step is imposed in the input signal but also amplitude and phase changes are also included. Under this situation the non linear behavior of the adaptive law clearly appears during the transient response and higher overshoots can be produced. This effect is shown in Fig. 10 where an input reference step from $100 \angle 0^{\circ}$ to $50 \angle-$ $30^{\circ}$ is done including a frequency step from $50 \mathrm{~Hz}$ to $45 \mathrm{~Hz}$ and backward. The results show that the proposed frequency adaptation loop works properly also under these conditions. Besides, the defined settling time is also achieved. It should be noticed that the transient response will vary for different amplitude or phase changes. This is because in (56) the values of $s p$ and $\tilde{v p}$ depend on the actual module and position of the input and output vectors. For the sake of simplicity, Fig. 11 only shows the output of the AVF for one phase. It can be observed that for increasing values of $T_{\omega}$ the AVF needs more time to reconstruct the input signal. Thus the trade-off between speed and overshoot should be considered in order to achieve a desired performance.

\section{AVF PERFORMANCE UNDER UNBALANCED INPUT CONDITIONS}

One of the most important tasks of a grid synchronization system is to obtain the positive- and negative-sequence of the grid voltage under unbalanced conditions. For this reason the scheme presented in Fig. 7 is tested when a fault occurs in the input voltage. The pre-fault voltage is $100 \angle 0^{\circ}$ with $50 \mathrm{~Hz}$ and the post-fault voltage is $50 \angle-30^{\circ}$ and $25 \angle 110^{\circ}$ for the positive- and the negative-sequence respectively. Besides, it has been also considered a sudden change in the grid frequency from $50 \mathrm{~Hz}$ to $45 \mathrm{~Hz}$. The design parameters are $k_{f}=0.73$ and $T_{\omega}=0.1 \mathrm{~s}$. Fig. 12 shows the obtained results. It can be noticed that the performance is not good. In Fig. 12a it can be observed that the estimated frequency does not achieve
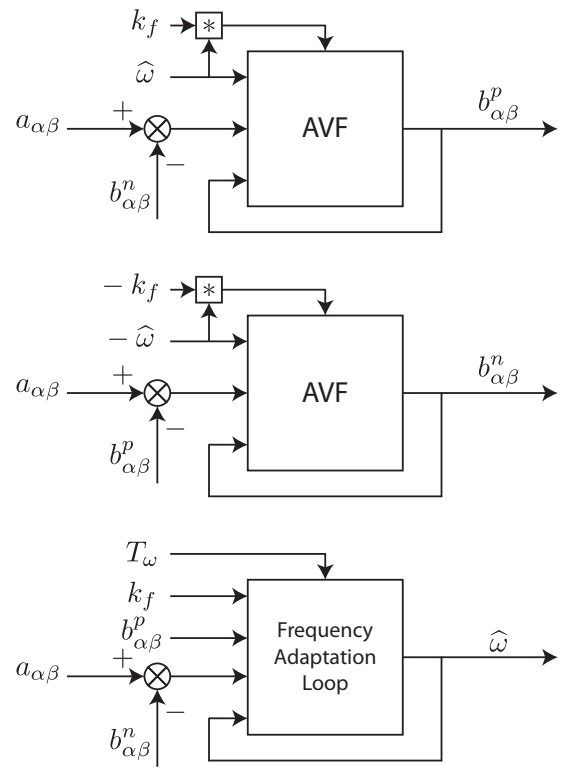

Fig. 13. Synchronization system scheme under unbalanced conditions.

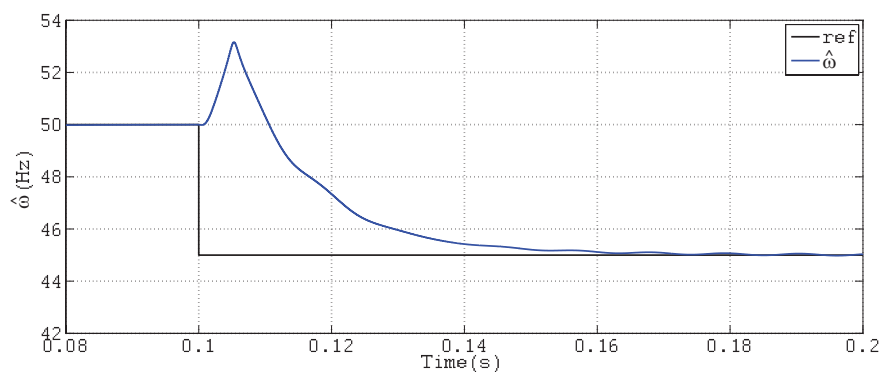

(a)

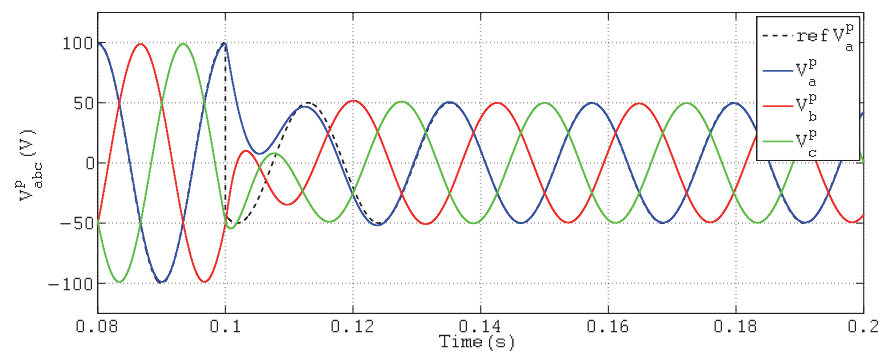

(b)

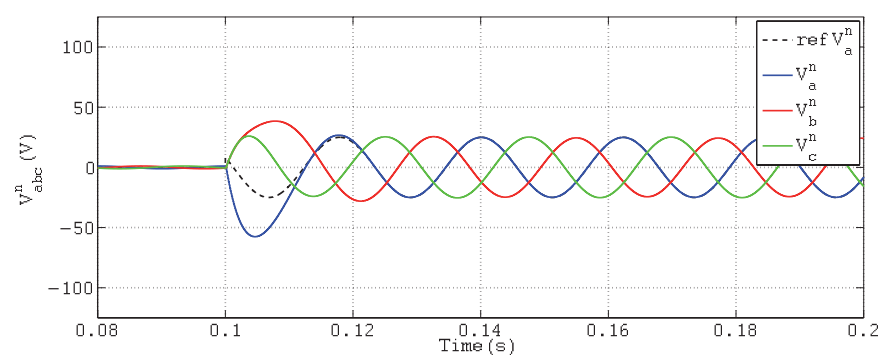

(c)

Fig. 14. System response: (a) Estimated frequency, (b) Positive output voltages, (c) Negative output voltages.

the actual input frequency value and moreover it has a non negligible ripple. On the other hand, Fig. 12b shows that the input positive-sequence is not reconstructed properly. Finally, 
this scheme does not allow to calculate the negative-sequence. For all these reasons, it is clear that it is necessary to modify the proposed synchronization scheme.

It was shown in section III that the AVF has unity gain and zero phase only for the tuned frequency. For any other frequency it attenuates the input. From Fig. 5 it can be observed that attenuation for other components depends on the value of design constant $k_{f}$. Therefore, if the input signal has several components, then one option will consist on decreasing the value of $k_{f}$ providing the desired attenuation. As a counterpart, lower values of $k_{f}$ imply higher settling times. As a consequence, this choice reduces the performance of the system and it should be avoided if possible. Besides, this solution does not solve the problem of reconstructing the negative-sequence of the input signal.

The proposed solution is to use two independent AVFs tuned for the positive- and negative-sequence respectively. Also, a cross-feedback network is also included. This network mainly consists on subtracting the undesired component at the input of each AVF. It should be noted that only one frequency adaptation loop is necessary because the frequency for the negative-sequence AVF can be calculated as $-\widehat{\omega}$. Besides, the inputs for the frequency adaptation loop are those corresponding to the positive-sequence signals. The scheme of the proposed solution is shown in Fig. 13. It should be noticed that structural differences between previous synchronization schemes and the proposed solution are present. For instance, the MSOGI-FLL requires a positive- and negative-sequence network calculation that is applied to the output signals from the SOGI-based filter [21]. The same approach is followed when ANFs are used [23], [24]. In the proposed AVF, it is needed the decoupling network before filtering the input signals due to the vectorial approach. Compared with other complex coefficient filter solutions, in [26] the decoupling network is not used, but a cascaded structure is proposed to extract the positive- and negative-sequence. On the other hand, the proposed cross-feedback network also appears in [28], in order to achieve the desired behavior.

Fig. 14 plots the obtained results under the previous fault conditions when the proposed modified synchronization scheme is used. Now it can be observed that the system performs as expected. In Fig. 14a is presented the estimated input frequency, as it can be noticed the steady state achieves the actual frequency value in the defined settling time. Regarding the positive- and negative-sequence, Fig. 14b and Fig. 14c display that both signals can be reconstructed properly maintaining the desired performance.

\section{SYNCHRONIZATION UNDER UNBALANCED AND DISTORTED INPUT CONDITIONS}

Unbalanced and distorted input voltages condition is a common situation after a grid fault. Following the same reasoning proposed in section VI, it is clear that it will be necessary to use one AVF for each harmonic and sequence that should be detected. Besides, the cross-feedback network is also included, thus the final synchronization scheme is shown in Fig. 15. It should be noticed that this kind of cross-feedback network
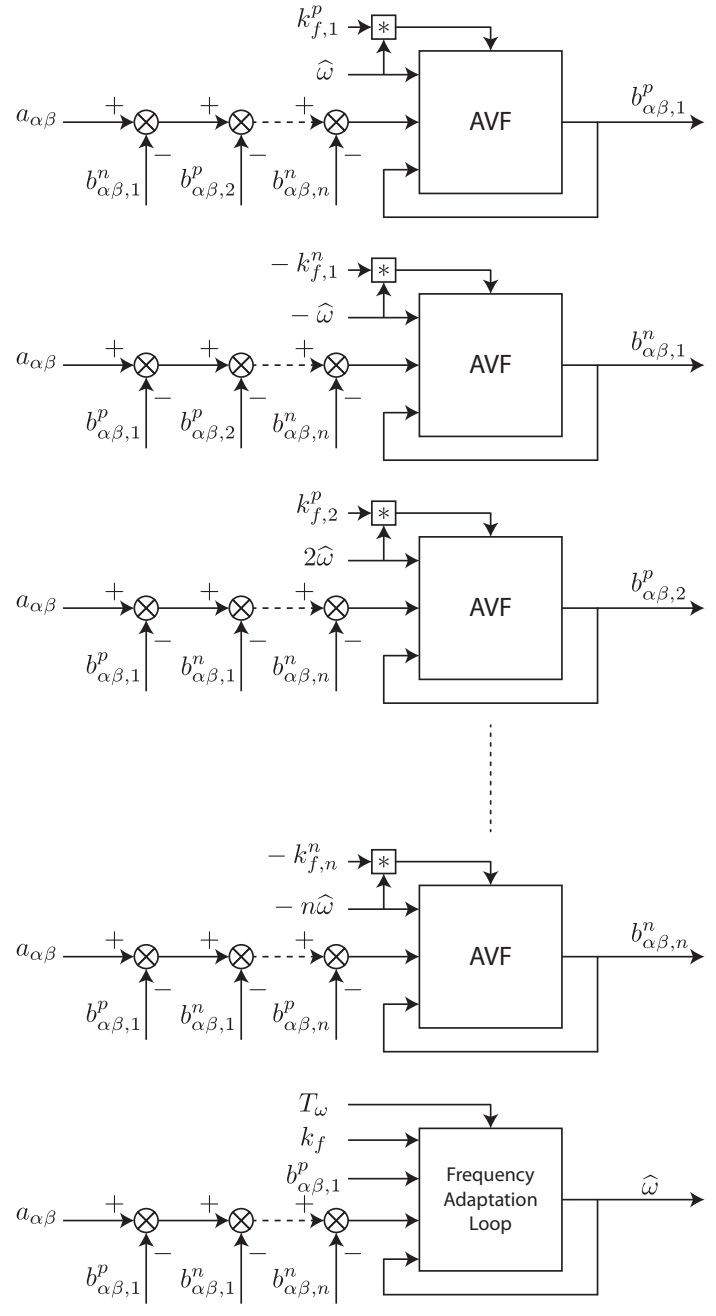

Fig. 15. Synchronization system scheme under unbalanced and distorted conditions.

TABLE I

SYSTEM VARIABLES.

\begin{tabular}{cc}
\hline \hline Parameter & Description \\
\hline $1^{\text {st }}$ harmonic positive-sequence & $50 \angle-30^{\circ}$ \\
$1^{\text {st }}$ harmonic negative-sequence & $25 \angle 110^{\circ}$ \\
$5^{t h}$ harmonic negative-sequence & $20 \angle 0^{\circ}$ \\
$7^{t h}$ harmonic positive-sequence & $20 \angle 0^{\circ}$ \\
$11^{t h}$ harmonic negative-sequence & $20 \angle 0^{\circ}$ \\
\hline \hline
\end{tabular}

has been previously used in other synchronization structures like in [28] or the MSOGI-FLL [21] where it was called harmonic detection network (HDN). This solution presented good results and for the proposed AVF scheme allows to remove the undesired harmonics components before the input signals are filtered.

The proposed scheme has been tested during a grid fault. Initially, the pre-fault grid voltage is $100 \angle 0^{\circ}$ with $50 \mathrm{~Hz}$. The post-fault voltage is composed by several harmonics with different sequences. Besides, it has been also considered a sudden change in the grid frequency from $50 \mathrm{~Hz}$ to $45 \mathrm{~Hz}$. The values for each harmonic are summarized in Table I and the resulting input voltages are plotted in Fig. 16. 


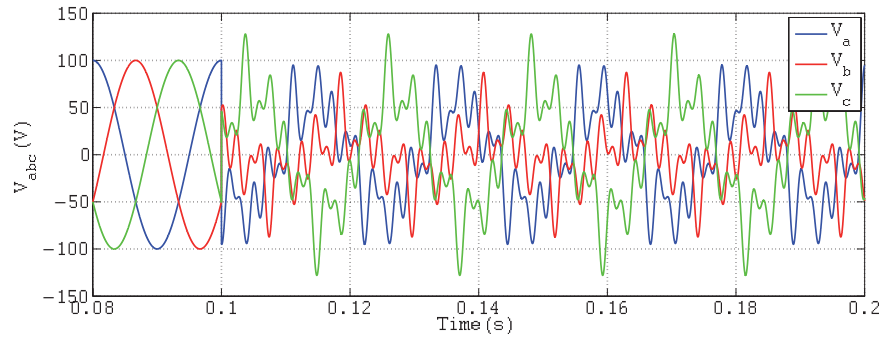

Fig. 16. Unbalanced and distorted input voltages for testing the proposed synchronization scheme.

To obtain the harmonic components of the input voltages five AVF have been used. Each one tuned to the appropriate harmonic and sequence, that is, positive $1^{\text {st }}$ harmonic, negative $1^{\text {st }}$ harmonic, negative $5^{\text {th }}$ harmonic, positive $7^{\text {th }}$ harmonic and negative $11^{\text {th }}$ harmonic. As the input voltages have high order frequency components, the sampling frequency has been set to $20 \mathrm{kHz}$ in order to obtain a more accurate reconstruction of the $11^{\text {th }}$ harmonic. It should be noticed that the number of samples in the reconstructed signal decrease when the harmonic order increases. The gains for the AVF tuned at the fundamental frequency have been set to $k_{f, 1}^{p}=0.73$ and $k_{f, 1}^{n}=0.73$. The gain for the rest of components are $k_{f, 5}^{n}=k_{f, 7}^{p}=k_{f, 11}^{n}=0.073$. This makes the AVFs for the high order harmonics more selective but does not diminish the performance of the system because the overall settling time is defined by the lower frequency component. Finally, the settling time for the frequency adaptation loop is set to $T_{\omega}=0.1 \mathrm{~s}$.

Fig. 17 shows the response of the proposed synchronization system under the aforementioned unbalanced and highly distorted grid conditions. The plot in Fig. 17a confirms that the frequency adaptation loop works properly and exhibits the expected behavior. Fig. 17b and Fig. 17c present the detection of the fundamental positive- and negative-sequences. It can be noticed that the signals are reconstructed almost perfectly. Finally, Fig. 17d, Fig. 17e and Fig. 17f display the behavior of the system for the reconstruction of the high order harmonic components. It can be observed that the proposed scheme provides and accurate detection of these components even when a step in the input frequency has been considered.

Finally, it should be noticed that the behavior of the MAVFFLL was tested without considering the effects of dc or interharmonics disturbances. However, it must be pointed out that the AVF attenuates the dc signal as can be observed from its bode diagram in Fig. 5. Besides, the MAVF-FLL is able to estimate inter-harmonics components as well, just by adding AVF blocks tuned at the proper frequency.

\section{EXPERIMENTAL RESULTS}

The behavior of the proposed synchronization algorithm has been tested in a real system. For this purpose a power converter prototype connected to a programmable three-phase ac source has been used. The experimental setup consists on a three-phase two-cell H-Bridge cascaded converter working as a STATCOM. The scheme of the laboratory setup is depicted in Fig. 18 and the main system parameters are summarized in Table II.

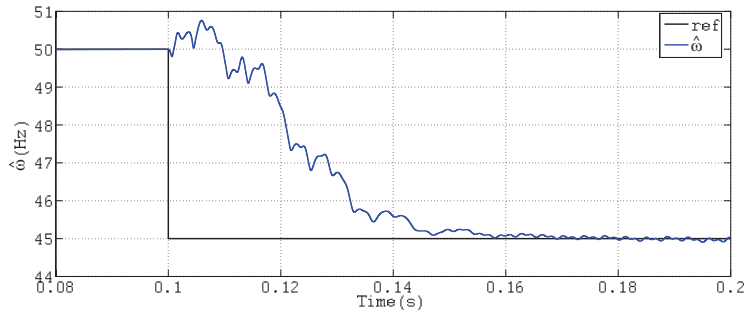

(a)

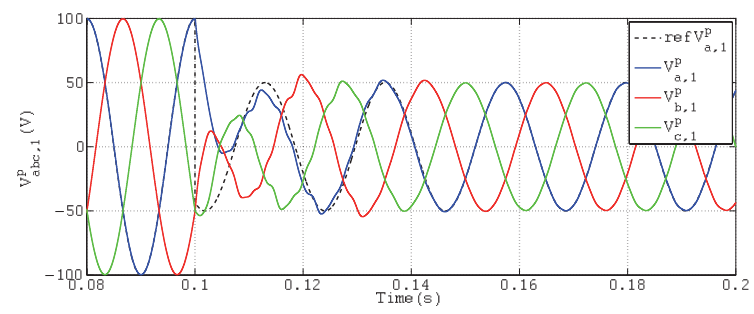

(b)

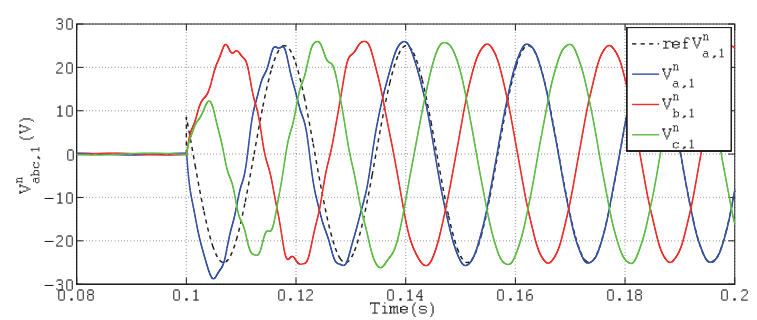

(c)

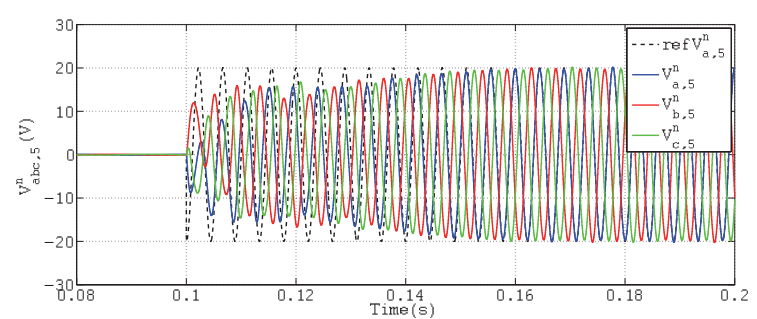

(d)

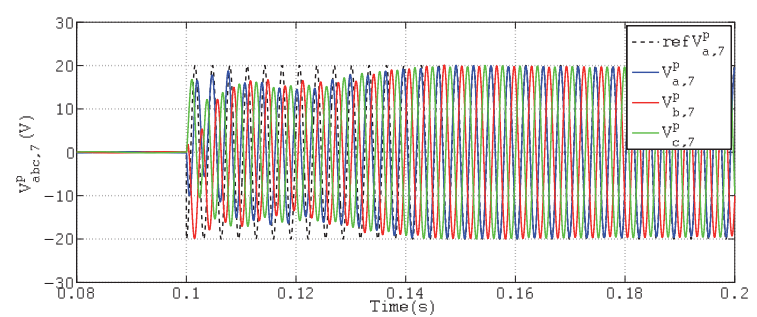

(e)

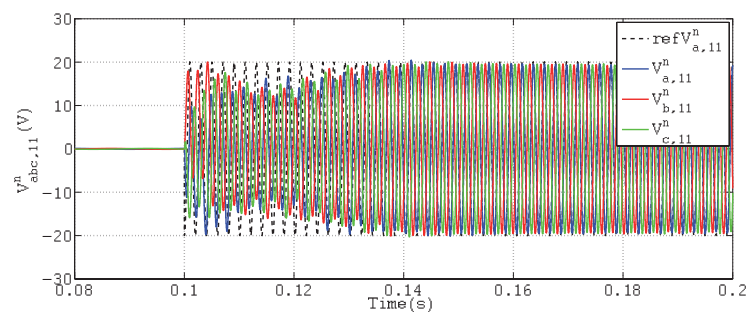

(f)

Fig. 17. Simulation system response: (a) Estimated frequency, (b) Positive $1^{\text {st }}$ harmonic output voltages, (c) Negative $1^{\text {st }}$ harmonic output voltages, (d) Negative $5^{\text {th }}$ harmonic output voltages, (e) Positive $7^{\text {th }}$ harmonic output voltages, (f) Negative $11^{t h}$ harmonic output voltages. 
Programmable

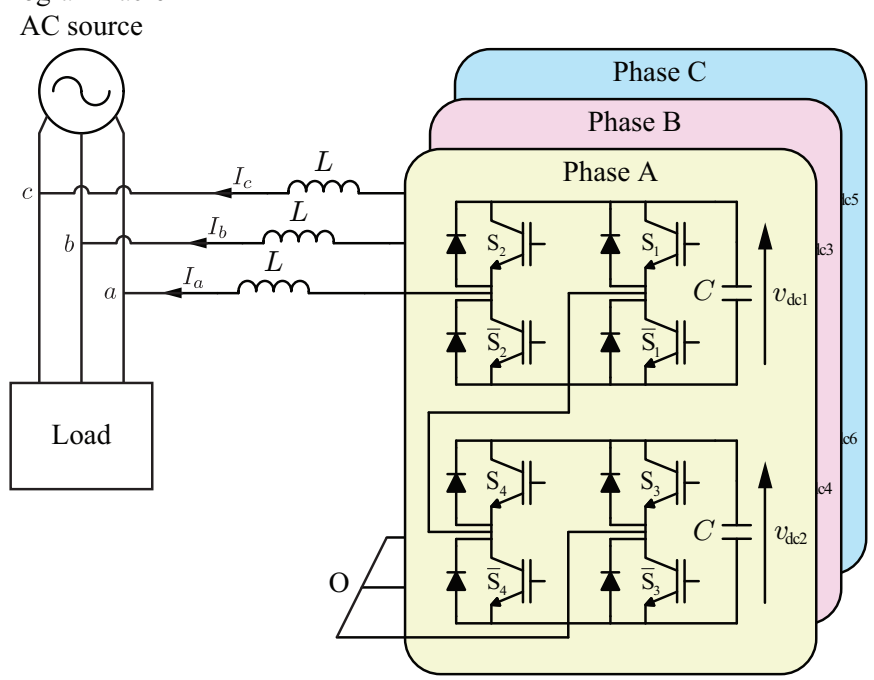

Fig. 18. Experimental setup layout.

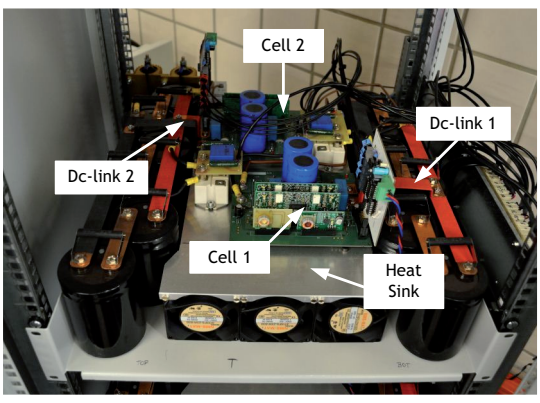

(a)

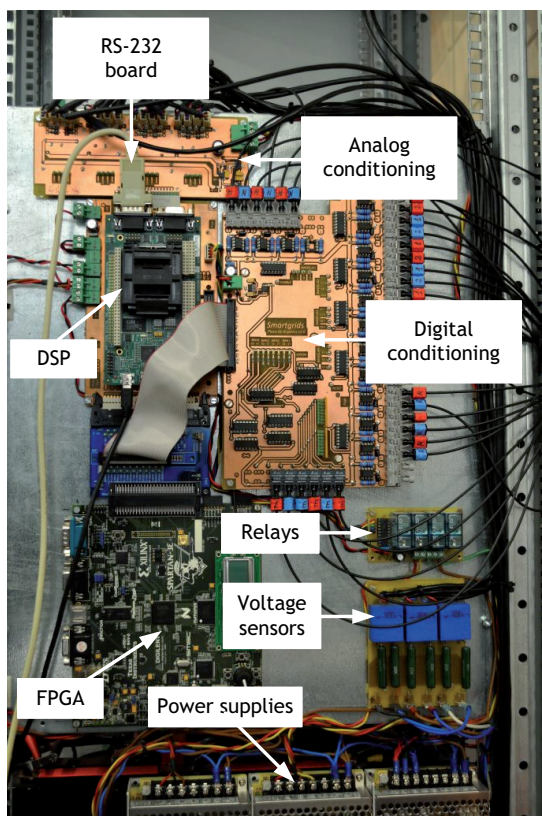

(b)

Fig. 19. Three-phase Two-Cell Cascade H-Bridge Converter laboratory prototype: (a) Power stage of one cell, (b) Hardware control boards.

The converter prototype is shown in Fig. 19. The core of the control hardware is based on the TMS320F28335 DSP
TABLE II

SYSTEM PARAMETERS.

\begin{tabular}{cc}
\hline \hline Parameter & Value \\
\hline Smoothing inductor & $L=1 \mathrm{mH}$ \\
DC-Link capacitor & $C=2200 \nu \mathrm{F}$ \\
Grid voltage (pre-fault) & $V=100 \mathrm{~V}$ \\
Control System & Operated on the TMS320F28335 DSP \\
Sampling frequency & $f_{s}=10 \mathrm{kHz}$ \\
\hline \hline
\end{tabular}

TABLE III

RESPONSE TIME AND THD.

\begin{tabular}{cccc}
\hline \multirow{2}{*}{ Harmonic } & \multicolumn{2}{c}{ Response Time } & \multirow{2}{*}{ THD } \\
& Theoretical & Experimental & \\
\hline $1^{\text {st }}$ positive & $54.1 \mathrm{~ms}$ & $66.3 \mathrm{~ms}$ & $0.57 \%$ \\
$1^{\text {st }}$ negative & $108.1 \mathrm{~ms}$ & $110.2 \mathrm{~ms}$ & $0.61 \%$ \\
\hline \hline
\end{tabular}

where the proposed MAVF-FLL has been coded using the bilinear transformation (Tustin) with pre-warping for its discrete implementation. During the experiments, there was no load connected and the power converter was not switching.Only the voltages at the point of common coupling where measured and the synchronization algorithm executed. The synchronization method has been tested under the same transient situation described in section VII. That is, it has been considered a pre-fault grid voltage of $100 \angle 0^{\circ}$ with $50 \mathrm{~Hz}$ and a postfault voltage composed by several harmonics with different sequences which are summarized in Table I. Besides, it has been also included a sudden change in the grid frequency from $50 \mathrm{~Hz}$ to $45 \mathrm{~Hz}$. The resulting input voltages in steady state are shown in Fig. 20.

Again, five AVF have been used, one for each harmonic and sequence component. This includes, the positive $1^{\text {st }}$ harmonic, negative $1^{\text {st }}$ harmonic, negative $5^{\text {th }}$ harmonic, positive $7^{\text {th }}$ harmonic and negative $11^{\text {th }}$ harmonic. The sampling frequency of the power converter prototype is $10 \mathrm{kHz}$, and the gains for the AVFs have been tuned to $k_{f, 1}^{p}=0.3, k_{f, 1}^{n}=0.15$ and $k_{f, 5}^{n}=k_{f, 7}^{p}=k_{f, 11}^{n}=0.1$. Finally, the settling time for the frequency adaptation loop is set to $T_{\omega}=0.1 \mathrm{~s}$.

To show the behavior of the proposed algorithm the internal variables of the DSP are shown in Fig. 21 and Fig. 22. The response of the proposed synchronization system under the aforementioned transient grid conditions is as expected from the simulation results. Fig. 21 confirms that the frequency adaptation loop works properly. The estimated frequency achieves its steady state value in approximately $100 \mathrm{~ms}$ as was defined in the settling time $T_{\omega}$.

Regarding the reconstruction of the different harmonic and sequence components, it can be observed in Fig. 22 that the algorithm also achieves good performance. Fig. 22a shows the input voltages measured with the DSP. It can be noted that the grid fault occurs when time is roughly $16 \mathrm{~ms}$. The detection of the fundamental positive- and negative-sequences are plotted in Fig. 22b and Fig. 22c respectively. It can be noticed that the signals are reconstructed almost perfectly. Table III shows the theoretical and experimental settling times and the THD of both waveforms. As can be seen, practical results fit correctly with analytical study. The slight difference 


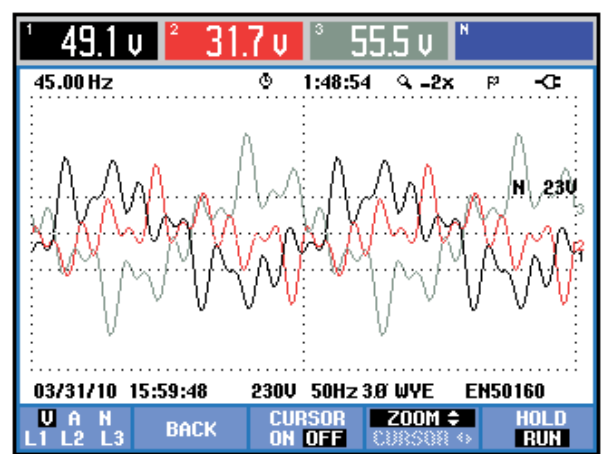

Fig. 20. Experimental steady state input voltages.

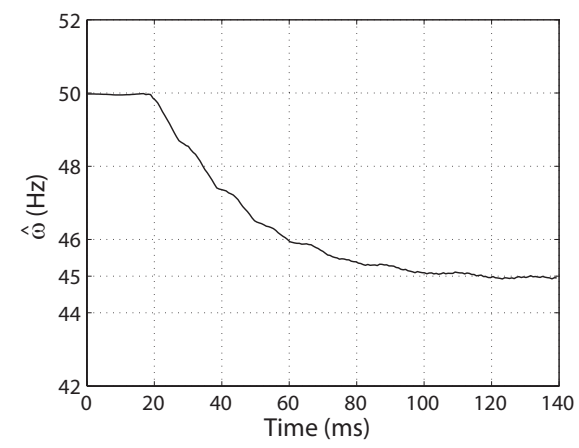

Fig. 21. Experimental transient response of the estimated frequency.

is due to the frequency adaptation loop, because the AVF was tuned considering $\omega$ as a constant or slowly variant value. A frequency step has been used in the experiment in order to show the robustness of the proposed algorithm but it is not usual in a real system. Regarding the THD values achieved they have been calculated using

$$
T H D=\sqrt{\frac{\sum_{k=2}^{\infty} s_{r m s, k}^{2}}{s_{r m s, 1}^{2}} .}
$$

The experimental THD can be considered as low, thus a good reconstruction of the fundamental positive- and negativesequences have been obtained. It should be noticed that speed and accuracy can be improved by using a higher sampling frequency, but due to hardware limitations in the laboratory prototype (low cost DSP) the computational burden has been restricted by choosing a suitable sampling frequency. Finally, Fig. 22d, Fig. 22e and Fig. 22f display the behavior of the systems for the reconstruction of the negative $5^{\text {th }}$ harmonic, positive $7^{\text {th }}$ harmonic and negative $11^{\text {th }}$ harmonic. It can be observed that the proposed scheme provides and accurate detection of these components.

\section{CONCLUSION}

Grid synchronization is a critical issue for power converters connected to the grid. It affects to the performance of the power and current control strategies used to manage the power converter. Besides, it is specially important for the integration of renewable energy sources where it is necessary to fulfill very demanding codes in order to have low-voltage ridethrough capability and remain connected during different grid faults. This paper presents a grid synchronization algorithm based on the use of vectorial adaptive filters. The proposed algorithm allows to estimate the grid frequency and the different harmonic sequence components independently of the harmonic and sequence content of the input voltage. The design of the whole algorithm has been presented in the paper and it has been addressed an important issue as the tuning procedure of the design constants in order to achieve a desired behavior of the system. In this way, it has been shown how the design constants should be chosen to define the settling time of the grid frequency estimation and the number of cycles needed to reconstruct the different harmonic sequence components. The algorithm has been tested with simulation and experimental results. In both cases have been proved that the algorithm performs correctly and it permits to estimate the grid frequency and calculate the different harmonic sequence values without regard to the input voltage harmonic sequence content. Moreover, it has been shown that the desired dynamic response is achieved and can be properly defined using the proposed tuning procedure for the design constants.

\section{REFERENCES}

[1] F. Blaabjerg, Z. Chen, and S. Kjaer, "Power electronics as efficient interface in dispersed power generation systems," Power Electronics, IEEE Transactions on, vol. 19, no. 5, pp. 1184 - 1194, sept. 2004.

[2] J. Carrasco, L. Franquelo, J. Bialasiewicz, E. Galvan, R. Guisado, M. Prats, J. Leon, and N. Moreno-Alfonso, "Power-electronic systems for the grid integration of renewable energy sources: A survey," Industrial Electronics, IEEE Transactions on, vol. 53, no. 4, pp. 1002 -1016, june 2006.

[3] S. Vazquez, S. Lukic, E. Galvan, L. Franquelo, and J. Carrasco, "Energy storage systems for transport and grid applications," Industrial Electronics, IEEE Transactions on, vol. 57, no. 12, pp. $3881-3895$, dec. 2010.

[4] Eltra and Elkraft. (2004) Wind turbines connected to grids with voltage below $100 \mathrm{kv}$. [Online]. Available: http://www.eltra.dk

[5] E.ON-Netz. (2006) Grid code-high and extra high voltage. GmbH. [Online]. Available: http://www.eon-netz.com/Ressources/downloads/ ENENARHS2006eng.pdf

[6] P. Rodriguez, A. Timbus, R. Teodorescu, M. Liserre, and F. Blaabjerg, "Flexible active power control of distributed power generation systems during grid faults," Industrial Electronics, IEEE Transactions on, vol. 54, no. 5 , pp. $2583-2592$, oct. 2007.

[7] S. Vazquez, J. Sanchez, J. Carrasco, J. Leon, and E. Galvan, "A modelbased direct power control for three-phase power converters," Industrial Electronics, IEEE Transactions on, vol. 55, no. 4, pp. 1647 -1657, april 2008.

[8] M. Malinowski, S. Stynski, W. Kolomyjski, and M. Kazmierkowski, "Control of three-level pwm converter applied to variable-speed-type turbines," Industrial Electronics, IEEE Transactions on, vol. 56, no. 1, pp. $69-77$, jan. 2009

[9] C. Yuen, A. Oudalov, and A. Timbus, "The provision of frequency control reserves from multiple microgrids," Industrial Electronics, IEEE Transactions on, vol. 58, no. 1, pp. $173-183$, jan. 2011.

[10] R. Portillo, S. Vazquez, J. Leon, M. Prats, and L. Franquelo, "Model based adaptive direct power control for three-level npc converters," Industrial Informatics, IEEE Transactions on, vol. 9, no. 2, pp. 11481157, 2013.

[11] M. Reyes, P. Rodriguez, S. Vazquez, A. Luna, R. Teodorescu, and J. Carrasco, "Enhanced decoupled double synchronous reference frame current controller for unbalanced grid-voltage conditions," Power Electronics, IEEE Transactions on, vol. 27, no. 9, pp. 3934 -3943, sept. 2012.

[12] C. Xia, M. Wang, Z. Song, and T. Liu, "Robust model predictive current control of three-phase voltage source pwm rectifier with online disturbance observation," Industrial Informatics, IEEE Transactions on, vol. 8 , no. 3 , pp. $459-471$, aug. 2012. 


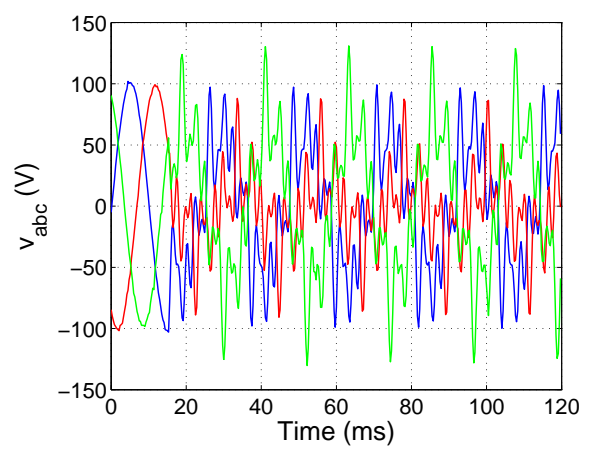

(a)

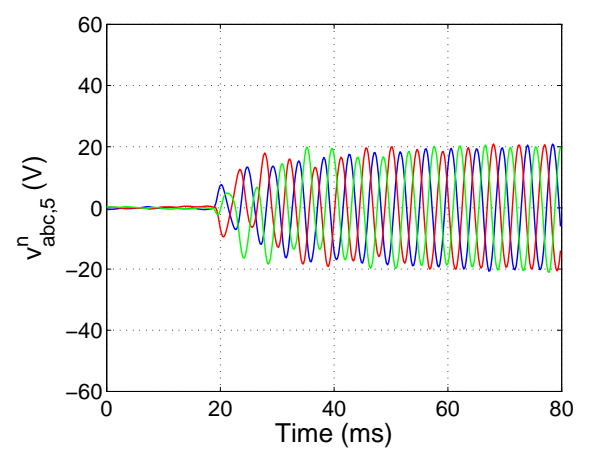

(d)

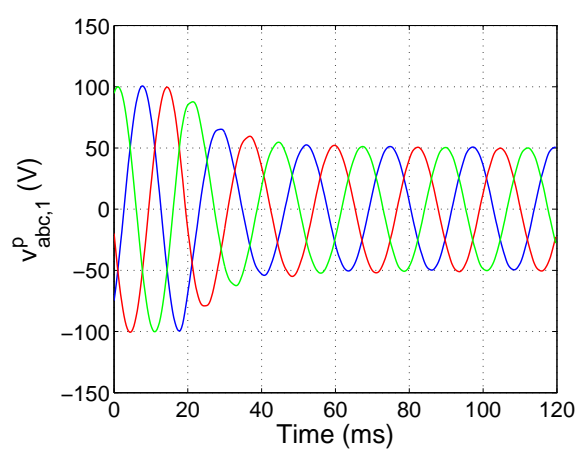

(b)

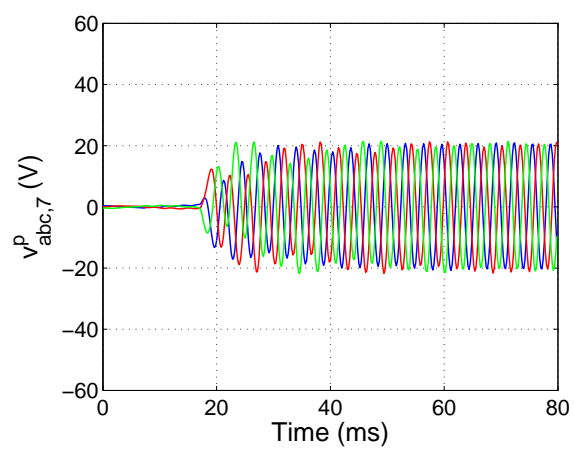

(e)

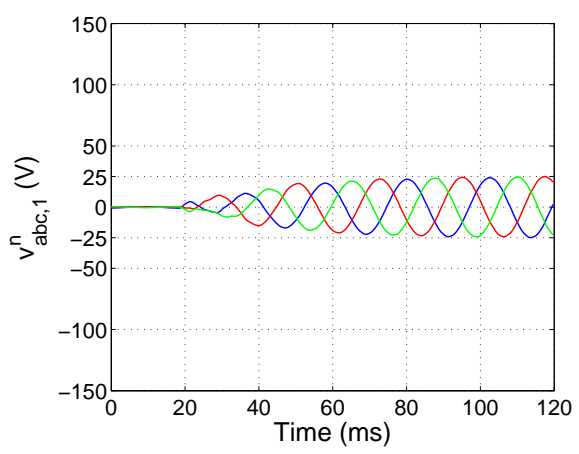

(c)

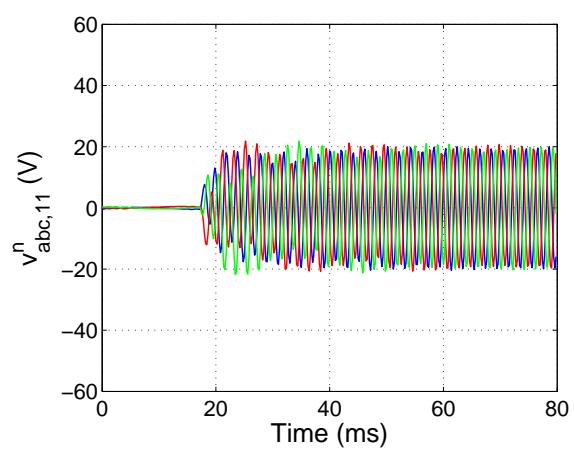

(f)

Fig. 22. Experimental transient response: (a) Input voltage, (b) Positive $1^{\text {st }}$ harmonic output voltages, (c) Negative $1^{\text {st }}$ harmonic output voltages, (d) Negative $5^{t h}$ harmonic output voltages, (e) Positive $7^{\text {th }}$ harmonic output voltages, (f) Negative $11^{\text {th }}$ harmonic output voltages.

[13] G.-C. Hsieh and J. Hung, "Phase-locked loop techniques. a survey," Industrial Electronics, IEEE Transactions on, vol. 43, no. 6, pp. 609 -615 , dec. 1996.

[14] V. Kaura and V. Blasko, "Operation of a phase locked loop system under distorted utility conditions," Industry Applications, IEEE Transactions on, vol. 33, no. 1, pp. $58-63$, jan/feb 1997.

[15] S. Shinnaka, "A robust single-phase pll system with stable and fast tracking," Industry Applications, IEEE Transactions on, vol. 44, no. 2, pp. $624-633$, march-april 2008.

[16] S.-K. Chung, "A phase tracking system for three phase utility interface inverters," Power Electronics, IEEE Transactions on, vol. 15, no. 3, pp. $431-438$, may 2000.

[17] F. Blaabjerg, R. Teodorescu, M. Liserre, and A. Timbus, "Overview of control and grid synchronization for distributed power generation systems," Industrial Electronics, IEEE Transactions on, vol. 53, no. 5, pp. $1398-1409$, oct. 2006.

[18] P. Rodriguez, J. Pou, J. Bergas, J. Candela, R. Burgos, and D. Boroyevich, "Decoupled double synchronous reference frame pll for power converters control," Power Electronics, IEEE Transactions on, vol. 22, no. 2, pp. $584-592$, march 2007.

[19] M. Karimi-Ghartemani, H. Karimi, and A. Bakhshai, "A filtering technique for three-phase power systems," Instrumentation and Measurement, IEEE Transactions on, vol. 58, no. 2, pp. 389 -396, feb. 2009.

[20] P. Rodriguez, A. Luna, M. Ciobotaru, R. Teodorescu, and F. Blaabjerg, "Advanced grid synchronization system for power converters under unbalanced and distorted operating conditions," in IEEE Industrial Electronics, IECON 2006 - 32nd Annual Conference on, nov. 2006, pp. $5173-5178$

[21] P. Rodriguez, A. Luna, I. Candela, R. Mujal, R. Teodorescu, and F. Blaabjerg, "Multiresonant frequency-locked loop for grid synchronization of power converters under distorted grid conditions," Industrial Electronics, IEEE Transactions on, vol. 58, no. 1, pp. $127-138$, jan. 2011.

[22] M. Mojiri, M. Karimi-Ghartemani, and A. Bakhshai, "Time-domain signal analysis using adaptive notch filter," Signal Processing, IEEE Transactions on, vol. 55, no. 1, pp. 85 -93, jan. 2007.

[23] D. Yazdani, A. Bakhshai, G. Joos, and M. Mojiri, "A real-time extraction of harmonic and reactive current in a nonlinear load for grid-connected converters," Industrial Electronics, IEEE Transactions on, vol. 56, no. 6, pp. $2185-2189$, june 2009.

[24] D. Yazdani, M. Mojiri, A. Bakhshai, and G. Joos, "A fast and accurate synchronization technique for extraction of symmetrical components," Power Electronics, IEEE Transactions on, vol. 24, no. 3, pp. $674-684$, march 2009.

[25] F. Neves, M. Cavalcanti, H. de Souza, F. Bradaschia, E. Bueno, and M. Rizo, "A generalized delayed signal cancellation method for detecting fundamental-frequency positive-sequence three-phase signals," Power Delivery, IEEE Transactions on, vol. 25 , no. 3, pp. $1816-1825$, july 2010.

[26] F. Wang, M. Benhabib, J. Duarte, and M. Hendrix, "High performance stationary frame filters for symmetrical sequences or harmonics separation under a variety of grid conditions," in Applied Power Electronics Conference and Exposition, 2009. APEC 2009. Twenty-Fourth Annual IEEE, feb. 2009, pp. $1570-1576$.

[27] X. Q. Guo, "Frequency-adaptive voltage sequence estimation for grid synchronisation," Electronics Letters, vol. 46, no. 14, pp. 980 -982, july 2010.

[28] X. Guo, W. Wu, and Z. Chen, "Multiple-complex coefficient-filterbased phase-locked loop and synchronization technique for three-phase grid-interfaced converters in distributed utility networks," Industrial Electronics, IEEE Transactions on, vol. 58, no. 4, pp. $1194-1204$, april 2011.

[29] M. Perales, J. Mora, J. Carrasco, and L. Franquelo, "A novel control method for active filters, based on filtered current," in Power Electronics Specialists Conference, 2001. PESC. 2001 IEEE 32nd Annual, vol. 3, june 2001, pp. $1408-1413$ vol. 3.

[30] J. Suul, A. Luna, P. Rodriguez, and T. Undeland, "Voltage-sensor-less synchronization to unbalanced grids by frequency-adaptive virtual flux estimation," Industrial Electronics, IEEE Transactions on, vol. 59, no. 7, pp. $2910-2923$, july 2012.

[31] H. K. Khalil, Nonlinear Systems, 3rd ed. Prentice Hall, 2002.

[32] W. Christiansen and D. Johnsen, "Analysis of requirements in selected grid codes," Tech. Rep., 2006. [Online]. Available: http: //www.frontwind.com/ 
[33] P. Rodriguez, A. Luna, R. Muñoz-Aguilar, I. Etxeberria-Otadui, R. Teodorescu, and F. Blaabjerg, "A stationary reference frame grid synchronization system for three-phase grid-connected power converters under adverse grid conditions," Power Electronics, IEEE Transactions on, vol. 27, no. 1 , pp. $99-112$, jan. 2012.

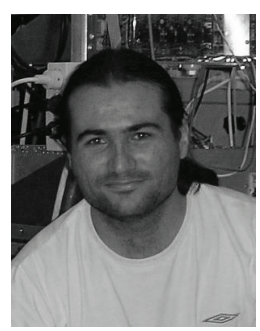

Sergio Vazquez (S'04, M'08) was born in Seville, Spain, in 1974. He received the B.S, M.S. and PhD degrees in industrial engineering from the University of Seville (US) in 2003, 2006, and 2010, respectively. In 2002, he was with the Power Electronics Group, US, working in R\&D projects. He is currently an Associate Professor with the Department of Electronic Engineering, US. His research interests include power electronics systems, modeling, modulation and control of power electronics converters applied to renewable energy technologies. Since 2008 he is co-founder of a technology based company called Win Inertia (www.wininertia.es). Within the industry and energy sectors Win Inertia is specialized in $\mathrm{R} \& \mathrm{D}$ of power electronics and control hardware technology applied to grid stabilization, electromobility, renewable energies integration and energy storage technologies. Dr. Vazquez was a co-recipient of the 2011 Best Paper Award of the IEEE TRANSACTIONS INDUSTRIAL ELECTRONICS.

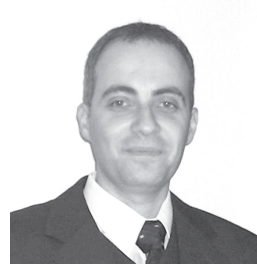

Juan A. Sanchez was born in Moron, Spain, in 1974. He received the B.S. and M.S. degrees in industrial engineering from the University of Seville (US), Seville, Spain, in 2001 and 2004 respectively. $\mathrm{He}$ is currently an Associate Professor with the University of Seville, where he is working pursuing his $\mathrm{Ph} . \mathrm{D}$ degree on the field of power electronics. His research interests include power active filters, power converters control and integration of renewable energy systems in the grid.

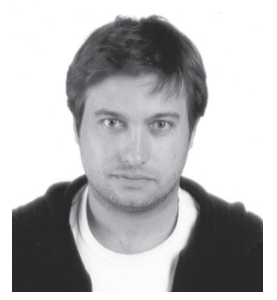

Manuel R. Reyes (S'06, M'11) was born in Seville, Spain, in 1981. He received the B.S., M.S. and $\mathrm{Ph} . \mathrm{D}$. degrees in telecommunications engineering from the University of Seville (US), Seville, Spain, in 2005, 2007 and 2012 respectively. From 2004 to 2012, he was with the Power Electronics Group, US, involving in $R \& D$ projects. He is currently working with the Power Electronics area at Win Inertia. His research interests include electronic power systems, flexible ac transmission systems, distributed power generation, and the integration of RES.

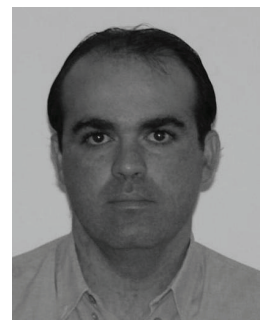

Jose I. Leon (S'04, M'07) was born in Cadiz, Spain, in 1976. He received the B.S., M.S., and $\mathrm{Ph} . \mathrm{D}$. degrees in telecommunications engineering from the University of Seville (US), Seville, Spain, in 1999, 2001 and 2006, respectively. Currently, he is an Associate Professor with the Department of Electronic Engineering, US. His research interests include electronic power systems, modulation and control of power converters, and industrial drives. Dr. Leon was co-recipient of the 2008 Best Paper Award of the IEEE INDUSTRIAL ELECTRONICS MAGAZINE and of the 2011 Best Paper Award of the IEEE TRANSACTIONS INDUSTRIAL ELECTRONICS.

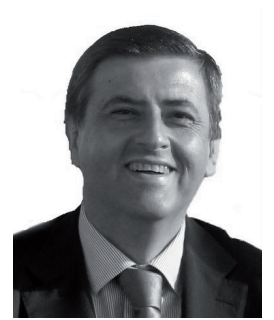

Juan M. Carrasco (M'97) was born in San Roque, Spain. He received the M.S. and Ph.D. degrees in industrial engineering from Seville University, Sevilla, Spain, in 1989 and 1992, respectively. He is currently Full Professor in the Department of Electronic Engineering of Seville University. He has been working for many years in the power electronic field where he was involved in a large number of industrial applications for the design and development of power converters for the energy sector. His current research area is Distributed Generation and especially he is working on the integration of renewable energy sources and FACTS converters for the contributing to the stability and the quality of the grid. He has been the Main Researcher for more than 70 technology industrial research projects with companies such as Abengoa, Endesa, Gamesa, ACS, ADIF, etc. Since 2002 he is co-founder of a technology based company called GPtech (www.greenpower.es). Within the energy sector GPtech is specialized in power electronic technology for the integration of renewable energies into the grid and FACTS converters. Professor Carrasco was a co-recipient of the 2011 Best Paper Award of the IEEE TRANSACTIONS INDUSTRIAL ELECTRONICS. 